\title{
Direct-Write of Nanoscale Domains with Tunable Metamagnetic Order in FeRh Thin Films
}

Cory D. Cress ${ }^{1 *}$, Darshana Wickramaratne ${ }^{2}$, Matthew R. Rosenberger ${ }^{3}$, Zachariah Hennighausen ${ }^{2}$, Patrick G. Callahan ${ }^{3}$, Samuel W. LaGasse ${ }^{4}$, Noam Bernstein ${ }^{3}$, Olaf M. van 't Erve', Berend T. Jonker', Syed B. Qadri', Joseph C. Prestigiacomo', Marc Currie ${ }^{5}$, Igor I. Mazin ${ }^{6}$, and Steven P. Bennett ${ }^{3 *}$

${ }^{1}$ Electronics Science and Technology Division, United States Naval Research Laboratory, Washington DC 20375, USA

${ }^{2} N R C$ Postdoc Residing at the Materials Science and Technology Division, United States Naval Research Laboratory, Washington DC 20375, USA

${ }^{3}$ Materials Science and Technology Division, United States Naval Research Laboratory, Washington DC 20375, USA

${ }^{4}$ NRC Postdoc Residing at the Electronics Science and Technology Division, United States Naval Research Laboratory, Washington DC 20375, USA

${ }^{5}$ Optical Sciences Division, United States Naval Research Laboratory, Washington DC 20375, USA

${ }^{6}$ Department of Physics and Astronomy and the Quantum Materials Center, George Mason University, Fairfax VA 22030, USA

*Corresponding Authors: cory.cress@nrl.navy.mil, steve.bennett@nrl.navy.mil 
In Figure S1 we show lattice parameter results from XRD as a function of temperature while cooling and heating through the transition. The lattice parameter was obtained by fitting the 003 peak shown in Figure 1(a) of the main text to a Gaussian and plotting the centroid. FWHM was also extracted from the Gaussian fits and converted to a crystallite size using the Scherrer equation and plotted as a function of temperature to show overall volume expansion of the FeRh lattice through the metamagnetic transition.
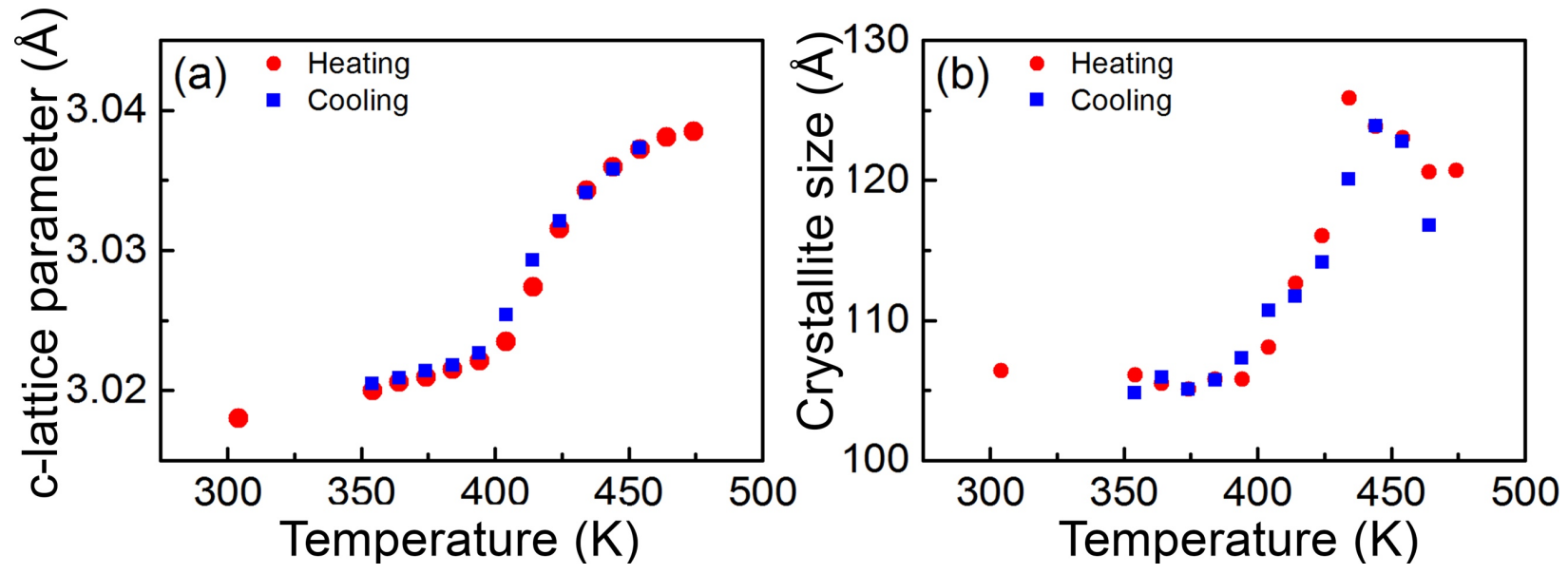

Figure S1. (a) Lattice parameter extracted from XRD of the 003 peak shown in Figure 1(a) of the main text as a function of temperature. (b) Crystallite size calculated by the Scherrer equation from the FWHM of the 003 peak as a function of temperature.

In Figure S2 we show a series of four AFMs taken for 4 different doses corresponding to the lowest and highest investigated in this study. Boxes 1-3 are in the regime of the lowest doses investigated in this work. In Box 1 and Box 2 a small increase in height is observed near the corner of the box whereas Box 3, the lowest dose, has no observable deviation. In contrast, the highest dose of $\sim 8 \times 10^{16} \mathrm{He}^{+} / \mathrm{cm}^{2}$, which exceeds all doses in this study, contains a region of apparent carbon deposition of $\sim 12 \mathrm{~nm}$ that is confined to the edge of the feature. Despite the lack of a height variation near the center of Box 10, we observe a clear contrast in the micrograph indicating that 
carbon deposition alone is not responsible for the changes we observe in the helium ion micrographs. Based on AFM micrograph analysis, the as-grown film RMS roughness is $\leq 1.5 \mathrm{~nm}$.

(a)
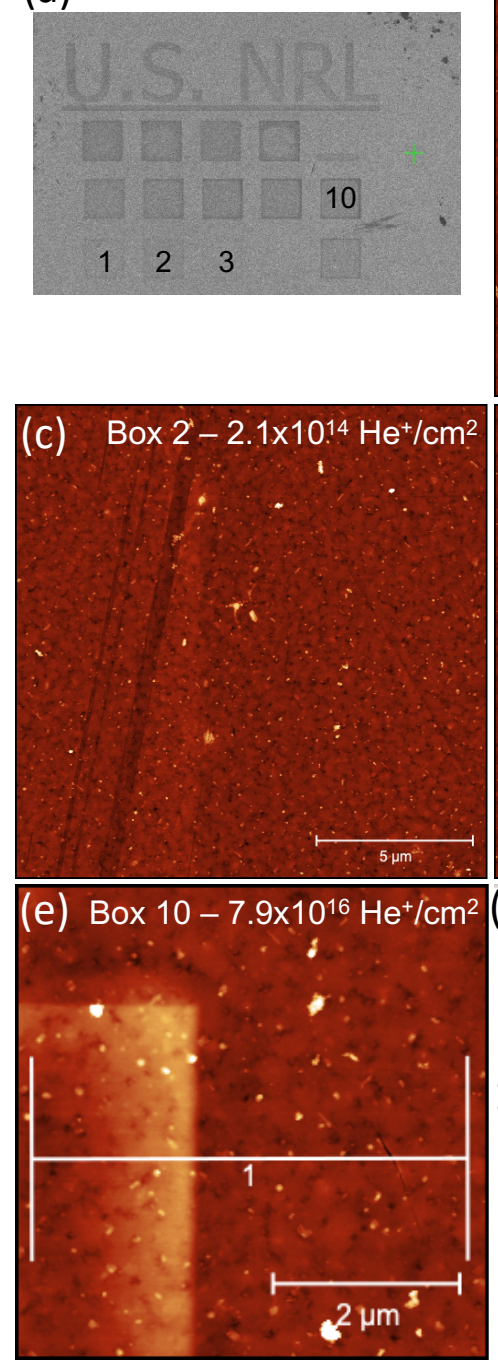
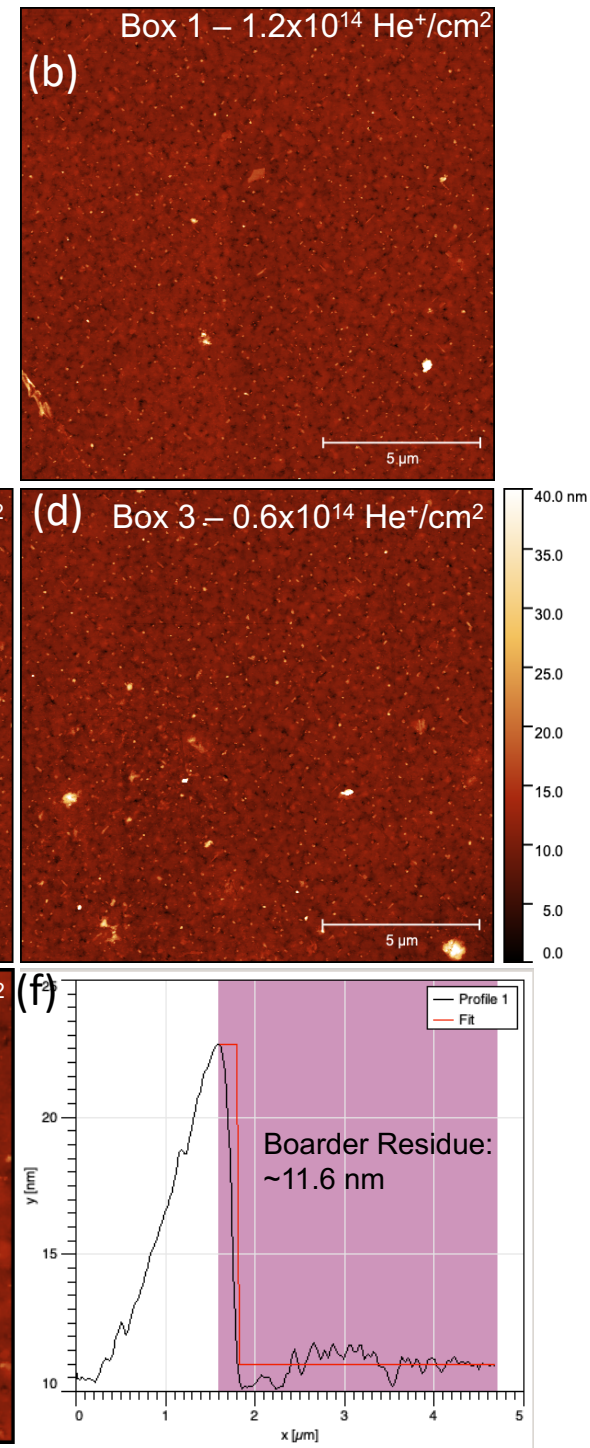

Figure S2. (a) Helium ion micrograph showing $20 \mu \mathrm{m} \times 20 \mu \mathrm{m}$ square dose array patterning, with box numbers labeled. (b-e) (Box 1, 2, 3, and 10) AFM height maps taken in the upper right corner of the boxes coinciding with the labels shown in (a). (f) AFM height cross section coinciding with scan length label " 1 " in (e) showing carbon residue deposit peak height of 11.6 $\mathrm{nm}$ from the highest dosed square, but is confined to the edge of the feature. 
In Figure $\mathrm{S} 3$ we overlay the $\mathrm{Fe}$ and $\mathrm{Rh}$ vacancies generated per $\mathrm{He}^{+}$per $\AA$ as a function of position within the film. These vacancy generation rates include the number of direct $\mathrm{He}^{+}$ion primary knock-ons, which is the number of atoms recoiling following a $\mathrm{He}^{+}$interaction, and the additional vacancies created by the $\mathrm{Fe}$ and $\mathrm{Rh}$ recoils. The maximum number of $\mathrm{He}^{+}$recoils occurs near $1000 \AA$, the center of the FeRh film and just prior to the projected $\mathrm{He}^{+}$range. The peak vacancy generation rate occurs before this depth (near $800 \AA$ ) because the energy of the Fe and Rh recoils is higher, enabling them to create a larger number of additional vacancies. The mean defect generation rate for $\mathrm{Fe}$ and $\mathrm{Rh}$ is approximately $0.052 \mathrm{Vac} / \AA \AA$ ion for each and the total number of vacancies generated per ion in a $2000 \AA$ FeRh (50/50) film is 212 vacancies. The bar graph overlaid in the figure illustrates the normalized final $\mathrm{He}^{+}$distribution after coming to rest in the film. The peak concentration of He, now neutralized, occurs at approximately $1100-1250 \AA$ into the film.

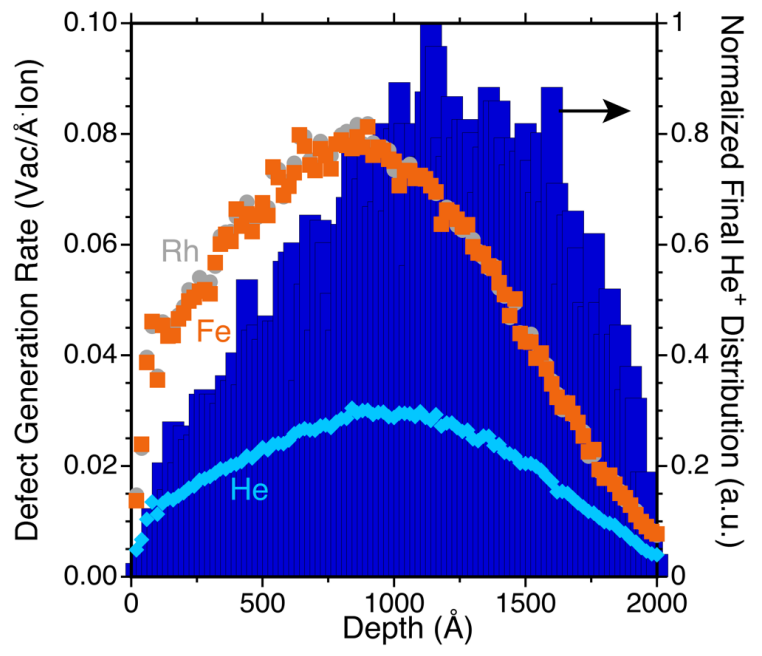

Figure S3. Data points (left y-axis): vacancy generation rate for $\mathrm{Fe}$ and $\mathrm{Rh}$ along with the number of $\mathrm{He}^{+}$induced primary knock-ons. Bar graph (right y-axis): normalized final He ion distribution in FeRh. 
Figure S4 contains MOKE microscopy images at $300 \mathrm{~K}$ and at $235 \mathrm{~K}, 150 \mathrm{~K}, 100 \mathrm{~K}$, and $7 \mathrm{~K}$, as the sample is cooled. At room temperature, all of the patterns except for the lowest doses along the first row (including $1.0 \times 10^{14}, 3.5 \times 10^{14}, 6.0 \times 10^{14}$, and $8.5 \times 10^{14} \mathrm{He}^{+} / \mathrm{cm}^{2}$ ) are apparent in the MOKE image. As the temperature is cooled, additional patterned regions from the MOKE image disappear beginning at $235 \mathrm{~K}$ and the trend continues down to $7 \mathrm{~K}$. These results confirm that the FeRh metamagnetic transition still occurs in the higher dosed samples, yet the transition occurs below room temperature. Therefore, even at these higher doses, sufficient magnetic order is preserved to induce the transition from ferromagnetic to antiferromagnetic at lower temperatures.

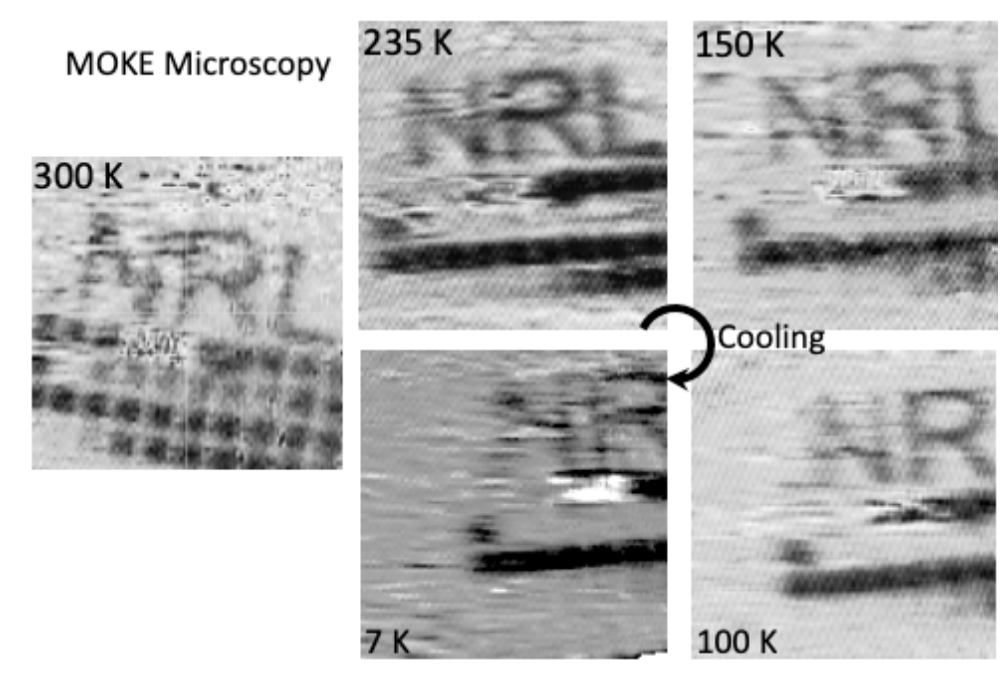

Figure S4. Temperature dependent MOKE imaging of a $\mathrm{He}^{+}$dose array pattern with temperatures labeled. The pattern consists of $20 \mu \mathrm{m} \times 20 \mu \mathrm{m}$ squares separated by $5 \mu \mathrm{m}$, with doses ranging from $1 \times 10^{14}$ to $2.3 \times 10^{15} \mathrm{He}^{+} / \mathrm{cm}^{2}$ along the first row and the second row ranging from $2.5 \times 10^{15}$ to $4.7 \times 10^{15} \mathrm{He}^{+} / \mathrm{cm}^{2}$. Contamination of the helium ion microscope tip occurred during patterning of the third row causing wide fluctuations in beam current making the dose for those features unknown. 
In Figure S5 we provide the abs(contrast) as a function of temperature from optical microscopy images during heating (red) and cooling (blue).

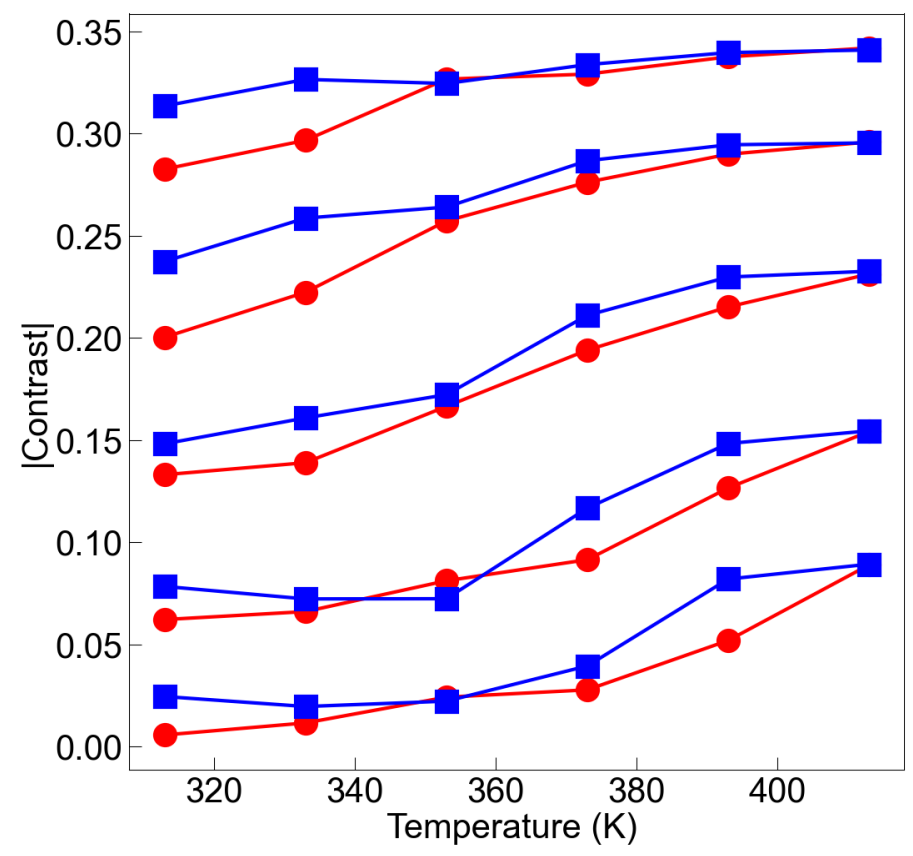

Figure S5. Optical contrast magnitude as a function of temperature for each image shown in Figure 4(a) of the main text. The red circles and blue squares correspond with images collected during heating and cooling, respectively. Starting from the bottom pair of lines the doses correspond to $1.1 \times 10^{14} \mathrm{He}^{+} / \mathrm{cm}^{2}, 2.2 \times 10^{14} \mathrm{He}^{+} / \mathrm{cm}^{2}, 4.0 \times 10^{14} \mathrm{He}^{+} / \mathrm{cm}^{2}, 6.2 \times 10^{14} \mathrm{He}^{+} / \mathrm{cm}^{2}$, and $8.0 \times 10^{14}$ $\mathrm{He}^{+} / \mathrm{cm}^{2}$. Each data set is offset by 0.05 units.

In Figure S6 we provide additional CAFM data of our nanoscale patterned structures. Figure S6(a) contains the height map corresponding with the current map from Figure 5(e) of the main text. It is apparent that there are approximately $1 \mathrm{~nm}$ depressions in the FeRh film corresponding with the location of each current peak. As noted in the text, the number of ions implanted into each spot corresponds with the total number of ions spread over the full area of the 
$25 \mathrm{~nm}$ features. Figure S6(b) contains a large area CAFM current map of the nanoscale feature arrays. At this scale, the $200 \mathrm{~nm}, 100 \mathrm{~nm}$, and $50 \mathrm{~nm}$ patterned regions are all clearly distinguishable within each of the square arrays. In Figure S6(c,d) we provide the AFM height and current maps, respectively, for the $25 \mathrm{~nm} / 50 \mathrm{~nm}$ square array. There is some correlation between height and current, yet in contrast to the spot array, many regions appear to be increased above the background height rather than showing a depression. The lateral dimensions are indeed close to the designed dimension of $25 \mathrm{~nm}$ on a $50 \mathrm{~nm}$ pitch (i.e., $25 \mathrm{~nm}$ spacing). In the vertical direction, the features are elongated and in some instances appear to touch. We suspect the origin of this is two-fold. First, the focus of the beam may not have been perfectly circular, slight elongation (i.e. astigmatism) along the y-axis would skew the feature shape. This would also explain the elongation along this direction for the spot array features. The second source of elongation may originate from the raster procedure of the NanoFab HIM. Following each point, the beam is electrostatically blanked before moving to the next point and repeating. If beam blanking occurs along the raster direction then it could be the source of additional ions. The fact that this occurs for the spot array and the rastered patterns likely points to beam focus / astigmatism as the primary cause. 
(a)

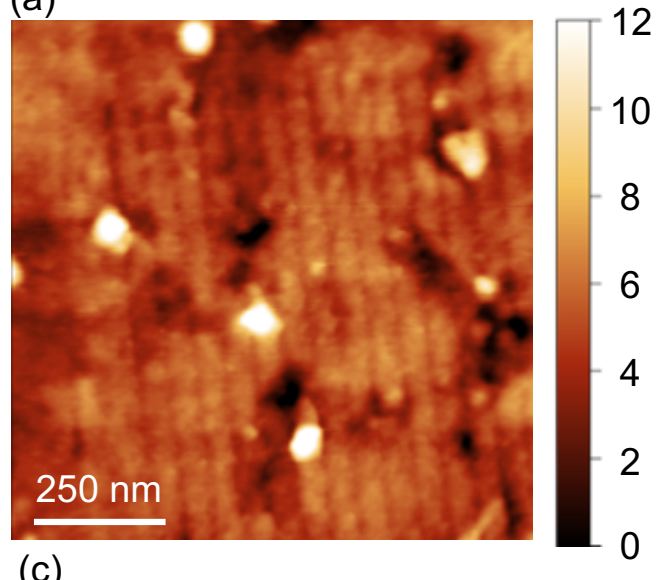

(c)

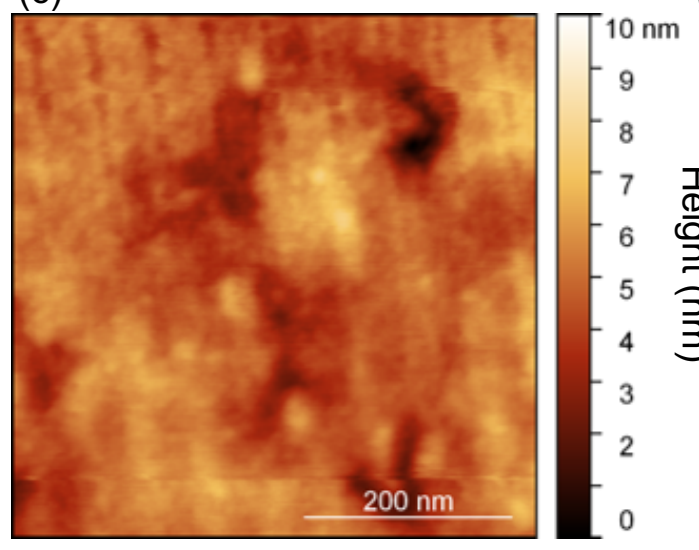

(b) $0 \mu \mathrm{m} \quad 2 \quad 4 \quad 6$

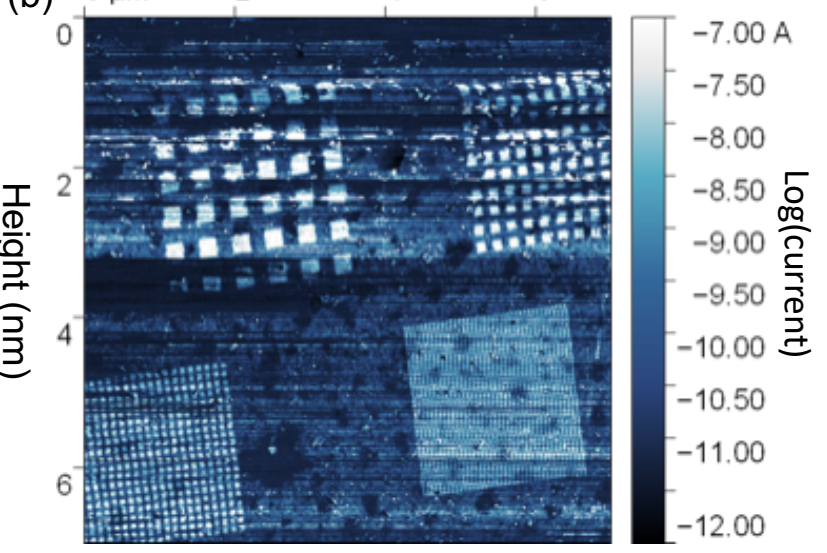

(d)

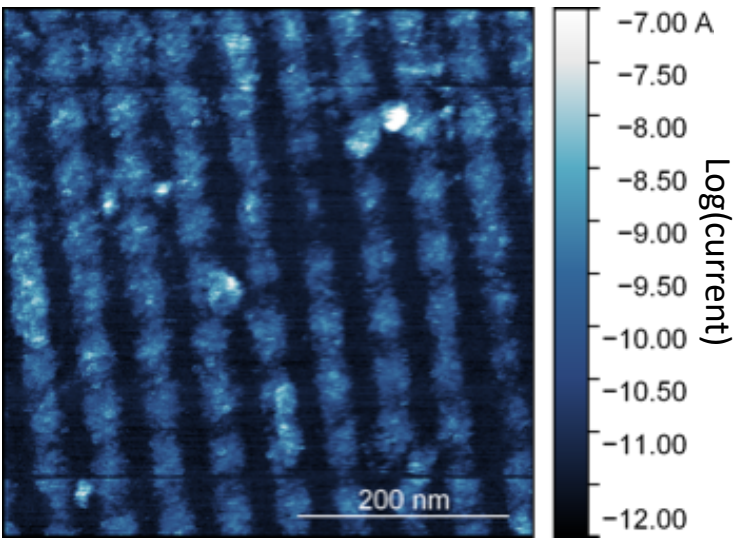

Figure S6. (a) AFM height map of the spot array sample corresponding with the current map from Figure 5(e) of the main text. (b) Log current map depicting regions of the four $2 \mu \mathrm{m} \times 2$ $\mu \mathrm{m}$ nanoscale feature arrays consisting of squares / pitches of: upper left $200 \mathrm{~nm} / 400 \mathrm{~nm}$, upper right $100 \mathrm{~nm} / 200 \mathrm{~nm}$, lower left $50 \mathrm{~nm} / 100 \mathrm{~nm}$, and lower right $25 \mathrm{~nm} / 50 \mathrm{~nm}$. (c) AFM height map and (d) current map of the array designed to have $25 \mathrm{~nm}$ squares spaced with a $50 \mathrm{~nm}$ pitch. 